\title{
Chaperone-like activities of the two pre-incubated small heat shock proteins of Bombyx mori
}

\author{
MT Hossain ${ }^{1 *}$ and Y Aso ${ }^{2}$ \\ Affiliation: ${ }^{1}$ Department of Biochemistry and Molecular Biology, Faculty of Agriculture, Bangladesh Agricultural \\ University, Mymensingh-2202, Bangladesh; ${ }^{2}$ Institute of Genetic Resources, Faculty of Agriculture, \\ Kyushu University, Fukuoka 812-8581, Japan
}

\begin{abstract}
The important small heat shock proteins (sHSPs) of Bombyx mori sHSP19.9, sHSP20.1, sHSP20.4, sHSP20.8, SHSP21.4 and SHSP23.7 are known to display distinct chaperone like activity (CLA) against a range of non-native protein aggregation during environmental stress. The small heat shock proteins sHSP19.9 and SHSP20.8 are identical polypeptides containing a single Cys residue: Cys-43 and Cys-123 respectively. The current information proposes that SHSPs function to prevent irreversible aggregation sometimes pre-incubation is pre-requisite to enhance their chaperone activities. In an attempt to determine their function, we have examined whether these two proteins have CLA using pre-incubation chaperone assay. The assay was conducted against the aggregation of a non-native protein, bovine liver catalase $(B L C)$, which is readily aggregated at $60^{\circ} \mathrm{C}$. Heat induced aggregation of BLC was decreased from 100 to $17 \%$ in the presence of pre-incubated SHSP20.8, which was $60 \%$ for without pre-incubation at a 1:0.5 molar ratio of BLC to SHSP. Whereas the aggregation was decreased from 100 to $33 \%$ in the presence of SHSP19.9 with dithiothreitol (DTT), which was $67 \%$ for without pre-incubation at a $1: 0.25$ molar ratio of BLC to SHSP. The functional reason for such variation might be due to the position of Cys residue in the amino acid sequence.
\end{abstract}

Key words: sHSPS, Pre-incubation, CLA, BLC, silkworm, sHSP19.9 and SHSP20.8

Bangladesh Animal Husbandry Association. All rights reserved. Bang. J. Anim. Sci. 2017. 46 (4):258-265

\section{Introduction}

Since exposure to high temperature represents a serious threat as at this condition the native proteins of living organism are unfolded and subsequently aggregated. The protein aggregates were toxic to the cells since they would impair the normal cellular functions (Horwich, 2002). The most documented sHSP of insect is that from Drosophila melanogaster composed of 4 main members (Hsp22, Hsp23, Hsp26 and Hsp27) and among them Hsp22 has been reported to increase its lifespan and resistance to oxidative stress (Arrigo, 2005, Morrow et al., 2006, Morrow et al., 2004). Eukaryotic small heat shock proteins (sHSPs) act as molecular chaperones by binding to denaturing proteins to form soluble complex and thus preventing their heat-induced aggregation (Kaldis et al., 2004; Horwich, 2002; Lee and Vierling 2000; Haslbeck et al., 1999). They are also believed to have involved for preventing protein aggregation under unfavorable conditions by blocking the hydrophobic surface exposed from the denaturing substrate proteins (Zhang et al.,
2005, Wang and Kurganov, 2003). The wheat HSP16.9 structure analysis predicts that several hydrophobic sites become exposed on disassembly of the oligomer into dimers (van Montfort et al., 2001). Thus it is reasonable to assume that the hydrophobic interface sites for the sHSP oligomer assembly are exposed due to its disassembly in response to heat stress and then become available for substrate binding, resulting in activation of the sHSPs (Hossain et al 2010). Although the silkworm, Bombyx mori has long been well-managed, well-documented, and used for biochemical and physiological research as a model organism, little information on the SHSP of this lepidopteran is available. $B$. mori has at least six genes encoding SHSP19.9, SHSP20.1, SHSP20.4, SHSP20.8, SHSP21.4 and sHSP23.7 (Aso et al., 2006 and Sakano et al., 2006). SHSP20.4 and SHSP21.4 have no Cys residues. SHSP23.7 has three Cys residues. Others have one Cys residue each (Sakano et al., 2006). SHSP20.8 and sHSP19.9 composed of identical polypeptides, and each of polypeptides has a single Cys residue: Cys-43 and Cys -123 respectively. The Cys residue of sHSP20.8 is in the $N$-terminal side of a-

*Corresponding author: mthossain@bau.edu.bd 
crystallin domain. This $N$-terminal side is very essential for substrate interactions and subsequently suppression of thermal aggregation of target protein (Basha et al., 2006). However, the conformation changes of both substrate and sHSPs are pre-requisite for correct interactions as well as to suppress the irreversible substrate aggregation by SHSPs. It is observed at lag-phase during incubation under stress (Bova et al., 2002). The investigation of exact active species of silkworm SHSP20.8 and SHSP19.9 was a matter of interest and so, the present study was conducted to observe the pre-incubation effects on the CLA of silkworm SHSP20.8 and SHSP19.9 against the aggregation of a nonnative protein, BLC.

\section{Materials and Methods}

\section{Materials}

Unless otherwise noted, the buffers used were $50 \mathrm{mM}$ sodium phosphate buffer ( $\mathrm{pH}$ 8.0) containing $0.1 \mathrm{M} \mathrm{NaCl}$ (buffer $A$ ), containing 0.3 $\mathrm{M} \mathrm{NaCl}$ (buffer B), and 20 mM HEPES buffer ( $\mathrm{pH}$ 7.7) containing $10 \mathrm{mM} \mathrm{NaCl}$ (buffer C). DTT was used as $10 \mathrm{mM}$. BLC was prepared with Milli Q $(2 \mathrm{~mm})$ water without further preparation. All of the used chemicals were reagent grade and freshly prepared. The incubation times were selected as 15, 20, 45, 60 and 240 minutes based on the requirement.

\section{Purification of sHSP19.9 and sHSP20.8}

SHSP20.8 and SHSP19.9 were prepared as recombinant $\mathrm{N}$-terminal His-tagged briefly described below.

\section{Pre-culture and culture of cells}

Each of sHSP19.9 and sHSP20.8 genes was sub-cloned into a pET28a expression vector. The resulting plasmids were introduced into the (DE3) E. coli cells and cultured at $37^{\circ} \mathrm{C}$ for 12 to 15 hour. Thus SHSP19.9 and SHSP20.8 were overproduced as recombinant proteins having $\mathrm{N}$-terminal His-tags. The entire procedure of purification was followed as described by Sakano et al. (2006) and Aso et al. (2007) with minor modifications (Hossain et al., 2010). The cells were grown up at $37^{\circ} \mathrm{C}$ on Luria-Bertani (LB) medium (Sigma, USA) until mid-log phase where absorbance at $600 \mathrm{~nm}$ was within 0.5 to 0.6 . After induction with $1 \mathrm{mM}$ Isopropyl $\beta-D-1$ thiogalactopyranoside (IPTG) (Wako Pure Chemical Industries Ltd. Tokyo, Japan), the cells were further cultured for 3 hour and collected from $150 \mathrm{ml}$ of LB medium by centrifugation at $1,095 \times \mathrm{g}$ for 20 minutes. The resulting cell pellet was made frozen with liquid nitrogen and stored at $-70^{\circ} \mathrm{C}$ until use.

\section{Isolation and purification of sHSPs from cells}

The pellet was suspended in $30 \mathrm{ml}$ of buffer $A$ and centrifuged at $1,095 \times \mathrm{g}$ for 20 minutes at $4^{\circ} \mathrm{C}$. The resultant pellet was re-suspended with $10 \mathrm{ml}$ of the same buffer and was lysed by sonication for 1 hour. Insoluble substance was removed by addition of $0.33 \mathrm{ml}$ of $5 \%$ polyethyleneimine (MP Biomedical Inc., Germany) and $1 \mathrm{ml}$ of $5.0 \mathrm{M} \mathrm{NaCl}$ and was stirred on ice for 1 hour and centrifuged at $17,528 \times \mathrm{g}$ for $20 \mathrm{~min}$. The resulting supernatant was taken, and soluble proteins were precipitated with $80 \%$ saturation of ammonium sulfate by stirring on ice for more than 1 hour. After centrifugation at 7,243 $\times \mathrm{g}$ for $20 \mathrm{~min}$, the resulting precipitate was suspended with buffer $\mathrm{C}$, kept at $4^{\circ} \mathrm{C}$ for 1 hour, and centrifuged at $7,243 \times \mathrm{g}$ for 20 minutes. The resulting supernatant was saturated with $80 \%$ of ammonium sulfate to precipitate proteins. It was done on ice for more than 1 hour and centrifuged at 7,243 $\times \mathrm{g}$ for 20 minutes. The resulting pellet was suspended with $10 \mathrm{ml}$ buffer $\mathrm{B}$ containing $45 \mathrm{mM}$ imidazole and poured into the suspension of Ni-NTA resin pre-equilibrated with the same buffer, and churned for 1 hour at $4^{\circ} \mathrm{C}$. The resin was washed with buffer B containing $45 \mathrm{mM}$ imidazole, and SHSPs were thus eluted with the buffer B containing $250 \mathrm{mM}$ imidazole. Four fractions ( $1 \mathrm{ml}$ each) were collected. Based on the higher absorbance at $280 \mathrm{~nm}$ of the eluted proteins, two fractions were mixed together and dialyzed overnight against buffer $\mathrm{C}$ at $4^{\circ} \mathrm{C}$.

\section{sHSP20.8 against BLC aggregation}

SHSP20.8 with buffer C was used against BLC aggregation in two conditions: with preincubation and without pre-incubation. Both time- and concentration-dependent CLA of SHSP20.8 were monitored by Shimadzu UV2400 double beam spectrophotometer at 360 $\mathrm{nm}$ wavelength. For the former case, $2.76 \mu \mathrm{M}$ sHSP20.8 was used against $8.96 \mu \mathrm{M}$ BLC and the activities were continuously monitored from 0 to 50 minutes. For the later one, different molar ratios; $1: 0$ (BLC $5.0 \mu \mathrm{M}), 1: 0.2,1: 0.5$, $1: 1$ and $1: 2$ were used in the cell and the performance was observed at $360 \mathrm{~nm}$.

The interaction between incubated BLC (at $60^{\circ} \mathrm{C}$ ) and SHSP20.8 at $60^{\circ} \mathrm{C}$ also was observed prior to investigate the CLA of pre-incubated SHSP20.8. BLC $(14.0 \mu \mathrm{M})$ was incubated for 5 and 7.5 minutes in the cell prior to addition of 
sHSP20.8 $(7.0 \mu M)$. The absorbance was monitored for 15 minutes. The entire process was repeated again at $30^{\circ} \mathrm{C}$ with the same conditions and methods. Moreover, BLC $(0.74$ $\mu \mathrm{M})$ was incubated at $60^{\circ} \mathrm{C}$ for $2,4,6,8,10$, 20 and 40 minutes and centrifuged at 10,955 $\times$ $\mathrm{g}$ for 5 minutes. The resultant supernatant was used again to monitor the interaction with 7.4 $\mu \mathrm{M}$ sHSP20.8.

Effects of pre-incubated SHSP20.8 on the CLA were monitored then. SHSP20.8 $(2.5 \mu \mathrm{M})$ with buffer $\mathrm{C}$ was incubated for different times; 5, 10,20 and 30 minutes prior to apply against BLC $(5.0 \mu \mathrm{M})$ aggregation with control (without pre-incubation). The used molar ratios for both cases were fixed at 1:0.5 (BLC and sHS20.8).

Concentration-dependent pre-incubation effects of SHSP20.8 were also observed using different molar ratios; 1:0 (control), 1:0.01, 1:0.05 and 1:0.1. SHSP20.8 and buffer $C$ (control). They were incubated for $30 \mathrm{~min}$ in the cell and fixed amount of BLC $(7.0 \mu \mathrm{M})$ was added to it. Keeping the molar ratio fixed at $1: 1$ but using higher $(40.0 \mu \mathrm{M})$ and lower concentration (5.0 $\mu \mathrm{M})$ of each sHSP20.8 and BLC, the CLA of 30 minutes pre-incubated SHSP20.8 was further monitored for 4 hours.

\section{sHSP19.9 against BLC aggregation}

By using the similar method as mentioned above, 30 minutes pre-incubated SHSP19.9 with $10 \mathrm{mM}$ DTT were used to observe the preincubation effects. The respective DTT containing sHSP19.9 with buffer C was incubated for 30 minutes and fixed amount of BLC $(5.0 \mu \mathrm{M})$ was added to it. The absorbance was taken at $360 \mathrm{~nm}$. Different molar ratios such as $1: 0.25,1: 0.5$ and $1: 0.75$ for BLC to sHSP19.9 were used to observe the effects of pre-incubation on CLA of SHSP19.9, whereas the same ratios were used as control (without pre-incubated).

\section{Data analysis and interpretation}

The used data were analysed and figures were prepared by using OriginPro7.5 Scientific Graphing and Analyzing Software (Origin Lab).

\section{Results}

To perform the chaperone assay of preincubated SHSPS, the SHSP19.9 and SHSP20.8 of $B$ mori were overexpressed in (DE3) E. coli cells with use of the corresponding pET28a expression vector and were purified through their $N$-terminal Histidine tag The yield of HissHSP (Histidine-tagged SHSP) was of the order of about $5 \mathrm{mg} / \mathrm{L}$ bacteria as recombinant protein as like Morrow et al., (2006).

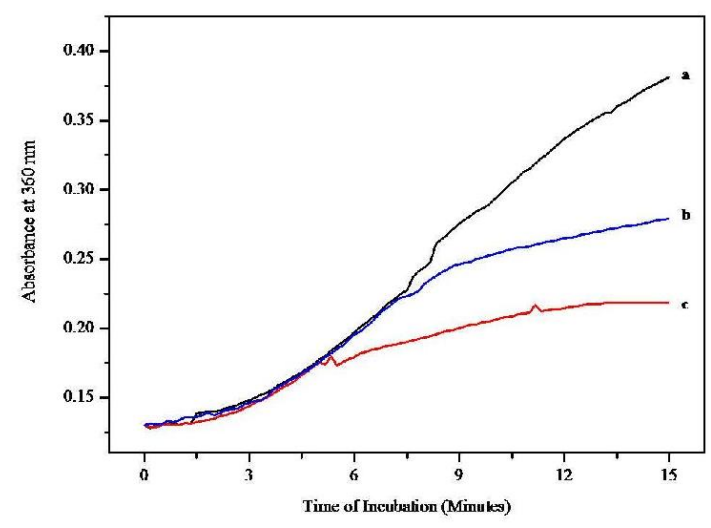

Figure 1. Effects of SHSP20.8 on the aggregation of $\mathrm{BLC}(14.0 \mu \mathrm{M})$ at $60^{\circ} \mathrm{C}$, where SHSP20.8 $(7.0 \mu \mathrm{M})$ was added after different times (b: $7.5 \mathrm{~min}, \mathrm{c}: 5.0$ $\min$ ) of BLC incubation

\section{Pre-incubated sHSP20.8 against BLC aggregation}

Because SHSPs of other organisms have been shown to have chaperone-like activity in vitro, we tested whether each of the sHSPs of $B$ mori could prevent protein denaturation and loss of function. So, prior to investigate the preincubation effects, sHSP20.8 was used against $B L C$ aggregation to observe its effects on the further aggregation of BLC. BLC was incubated at $60^{\circ} \mathrm{C}$ for 15 minutes alone and then SHSP20.8 was added after 5 and 7.5 minutes of BLC incubation. Result from presented figure 1 revealed that aggregation of BLC was partially halted by addition of HSP20.8, indicating that already aggregated BLC cannot be solubilized as well as renatured by SHSP20.8.

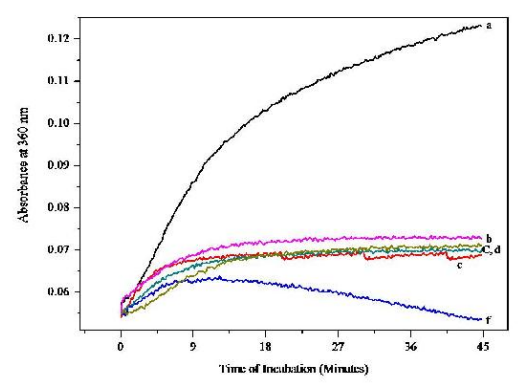

Figure 2. Pre-incubation effects on the CLA of SHSP20.8, where the activities show with fixed 1:0.5 molar ratio for BLC $(5.0 \mu \mathrm{M})$ to SHSP20.8 at different times of incubation: 0 (c), 5 (b), 10 (d), 15 (e) and 20 (f) $\mathrm{min}$. 
Next we determined the time dependent chaperone-like activity of sHSP20.8 against BLC. The molar ratio for BLC to SHSP20.8 was kept fixed at $1: 0.5$. It was observed that among the various times of incubation of SHSP20.8, incubation with 20 minutes is the best performer compare to others (Figure 2). The figure also revealed that pre-incubation of sHSP20.8 (f) has better activity than the without pre-incubated (b) against BLC aggregation.

Similar experiment was done by using SHSP20.8-BLC mixture at different molar ratios to observe the concentration dependent chaperone like activity with fixed pre-incubation time. The incubation time was fixed for 30 minutes. Using the same molar ratios, preincubated and without pre-incubated sHSP20.8 was applied against BLC. It was observed that fixed 1:0.05 molar ratio ( $f$ ) for BLC to SHSP20.8 shows the better performance than others and also than the without pre-incubation ratios (Figure 3). So, it can be mentioned that preincubation bears reproducible effects than the without pre-incubation, which is similar to the chaperone of other organisms that preincubation is an enhancing factor for CLA.

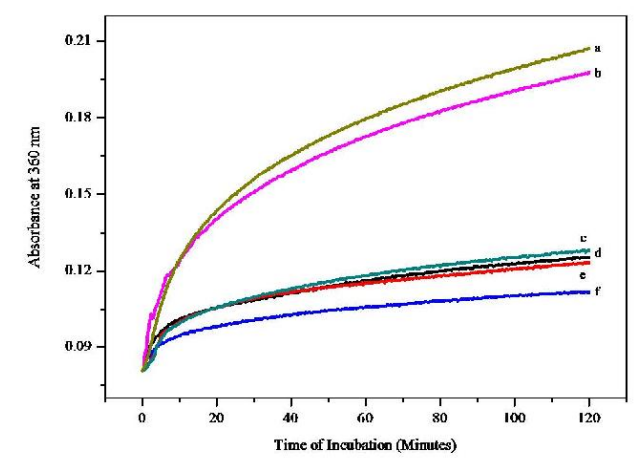

Figure 3. CLA of $30 \mathrm{~min}$ pre-incubated sHSP20.8 with 1:0.01 (a), 1:0.05 (f) and $1: 0.1$ (d) molar ratios along with control (without pre-incubated SHSP20.8) 1:0.01 (b), 1:0.05 (c) and 1:0.1 (e) molar ratios of catalase $(7.0 \mu \mathrm{M}$ fixed) to SHSP20.8

Various concentrations of pre-incubated sHSP20.8 were used against fixed $25.0 \mu \mathrm{M}$ BLC, and CLA was measured. Although marked differences between the activities of preincubated and without pre-incubated sHSP20.8 were not evident, the molar ratio 1:0.05 molar ratio for BLC to pre-incubated SHSP20.8 shows a little bit better performance (Figure 4).

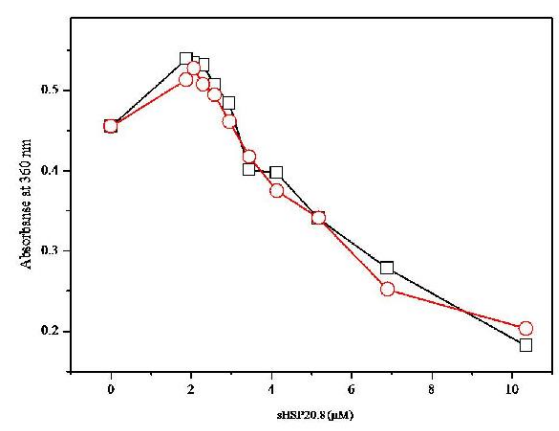

Figure 4. Pre-incubated SHSP20.8 against BLC aggregation, where the activities with ( $\square$ ) and without pre-incubated (0) SHSP20.8 is represented (from 0 to above $10.0 \mu \mathrm{M}$ ) against $25.0 \mu \mathrm{M}$ BLC.

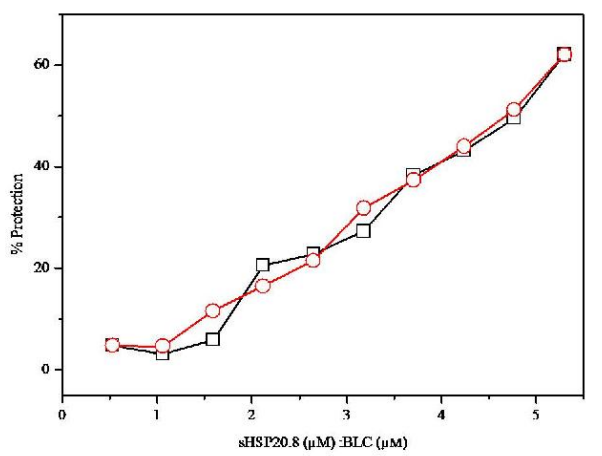

Figure 5. CLA of pre-incubated SHSP20.8. Protection (\%) of BLC aggregation by SHSP20.8 with ( $\square$ ) and without preincubated (o) SHSP20.8 at fixed 1:1 molar ratio is shown here

Independent performances monitored during determination of BLC aggregation protection (\%) by SHSP20.8 is presented in Figure 5. Keeping fixed molar ratio for sHSP20.8 and $B L C$, the mixture containing their larger amounts were used for studying the preincubation effects of SHSP20.8. In observation of long time CLA, the pre-incubated curve showed a slight halt compared to the without pre-incubated SHSP20.8 indicating the evidence of the positive performance of pre-incubation of the protein against the irreversible aggregation of BLC (Figure $6 \& 7$ ). This finding is alike with the finding of Smýkal et al., (2000) for tobacco HSP18 against citrate synthase aggregation. 


\section{Pre-incubated sHSP19.9 against BLC aggregation}

sHSP19.9 is aggregated at low ionic strength (buffer C) but in the presence of $10 \mathrm{mM} \mathrm{DTT}$, such aggregation was not evident (Hossain and Aso, 2017). So, the aggregation suppressive activity of pre-incubated SHSP19.9 was observed against BLC aggregation at $60^{\circ} \mathrm{C}$ in the presence of DTT. To observe effects of the pre-incubation on the CLA of SHSP19.9, $10 \mathrm{mM}$ DTT containing different concentrations of sHSP19.9 was pre-incubated prior of addition to BLC.

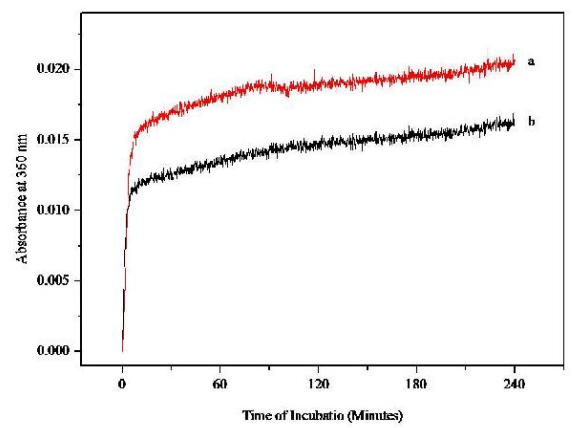

Figure 6. CLA of $30 \mathrm{~min}$ pre-incubated and without pre-incubated SHSP20.8 at fixed $1: 1$ molar ratio with lower concentration (5.0 $\mathrm{MM}$ each)

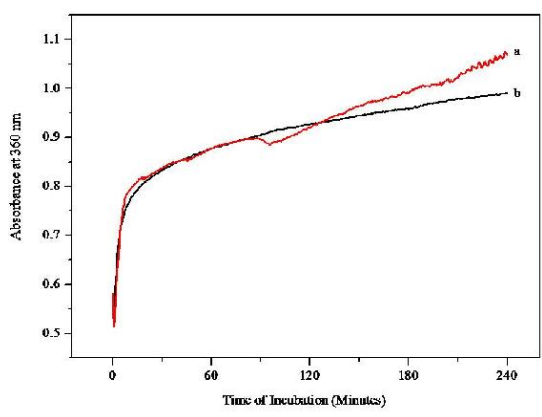

Figure 7. CLA of $30 \mathrm{~min}$ pre-incubated and without pre-incubated SHSP20.8 at fixed $1: 1$ molar ratio with higher concentration (40.0 $\mu \mathrm{M}$ each) molar ratio

A wide ranged concentration series of $B L C$ and sHSP19.9 was used with control for each ratio (Figure 8A-D). Results showed that all of the used concentration ratios are positive for preincubated SHSP19.9. Although the performance of pre-incubated and without pre-incubated sHSP19.9 were not drastically different, the pre-incubated SHSP19.9 at molar ratio $1: 0.25$ molar ratio for BLC to SHSP19.9 showed a little better performance than the control. So it becomes evident that pre-incubation had also some extent of significant effect on CLA of sHSP19.9.

\section{Discussion}

Despite reports that many sHSPs from yeast, plants and mammals present chaperone-like activity in in vitro aggregation assay, their function in vivo remain unknown. Overexpression of silkworm sHSP19.9 and SHSP20.0 has been shown to confer resistance to supraoptimal temperatures and also showed chaperone-like activity to inhibit heat-induced aggregation of BLC (Hossain et al., 2010). Thus these two silkworm sHSPs perform functions similar to sHSPs of other organisms in vitro chaperone assay (Panesenko et al., 2002; Abdulle et al., 2002; Van Montfort et al., 2002). But the exact mechanism of the chaperone action is not completely known but it is now evident that temperature regulated exposed of hydrophobic surface and temperaturedependent reversible dissociation of oligomers into smaller subunits such as dimers (van Montfort et al., 2001; Haslbeck et al., 1999) are involved in the chaperone mechanism of sHSPs. Changes in quaternary structure at temperatures that are physiologically relevant for sHSP are important for their chaperone activity (Van Montfort et al., 2002; Haslbeck et al., 1999; Giese and Veirling, 2002). Moreover, sometimes the pre-incubation is the prerequisite for the aggregation prevention mechanism (Manna et al., 2001). To understand the active species as well as the factors influence chaperone activities, we incubated the SHSP19.9 and SHSP20.8 prior to load against BLC. The performance was monitored for various times of incubation according to necessity. Although the two silkworm sHSPs share a high degree of homology, they display differences in their capacity to prevent aggregation of a common substance, BLC, suggesting difference in their mode of action. SHSP20.8 was more efficient than SHSP19.9 and solely can prevent the aggregation whereas SHSP19.9 required DTT to tolerate heat stress and to show the CLA as well (Hossain and Aso, 2017). This difference is not caused by their mode of preparation, as demonstrated with His-tagged purified under native condition. SHSPs of other organisms have also been reported to show differences intheir chaperone activities; mammalian Hsp22 
(Chowdary et al., 2004) and S. cerevisiae Hsp42 and Hsp26 (Haslbeck 2004).

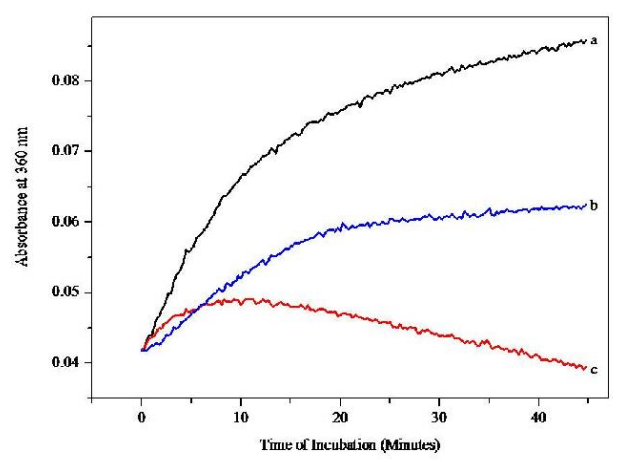

Figure 8A. Pre-incubated SHSP19.9 (at $60^{\circ} \mathrm{C}$ for $30 \mathrm{~min}$ ) with $10 \mathrm{mM}$ DTT against BLC aggregation with 1:0.25 concentration ratios, where (a) represents BLC only, (b) and (c) show without and with preincubated SHSP19.9 respectively.

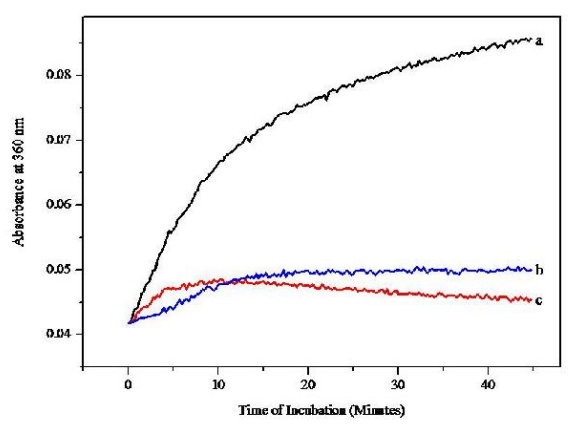

Figure 8B. Aggregation suppressive activity of pre-incubated sHSP19.9 (for $30 \mathrm{~min}$ at $60^{\circ} \mathrm{C}$ with $10 \mathrm{mM}$ DTT) against BLC aggregation with 1:0.5 concentration ratio, where (a) represents BLC only, (b) and (c) indicate without and with pre-incubated sHSP19 respectively.

Among the various substrate proteins, BLC is seen to be rapidly inactivated upon incubation at $60^{\circ} \mathrm{C}$, and about $80 \%$ of original activity is lost within 10 min (Hook and Harding, 1997). So it was used and the performance of preincubated silkworm sHSP20.8 and sHSP19.9 (with DTT) on the aggregation reaction of BLC was examined. When the silkworm sHSPs were added the aggregation of BLC was suppressed and pre-incubation bears reproducible effects. Such finding is also evident for Hsp16.3. Hsp16.3 pre-heated at high temperature was reported to exhibit significantly higher chaperone activity (Mao et al., 2001; Xinmiao et al., 2003). Therefore, the effects of preincubation of SHSP20.8 on its CLA were examined.

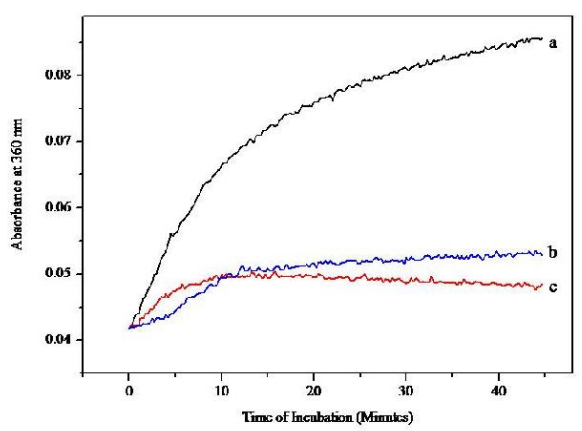

Figure 8C. Pre-incubated sHSP19.9 (for 30 min) with $10 \mathrm{mM}$ DTT against BLC aggregation at $60^{\circ} \mathrm{C}$ with $1: 0.75$ molar ratios, where (a) represents BLC only, (b) and (c) demonstrate without and with preincubated SHSP19 respectively.

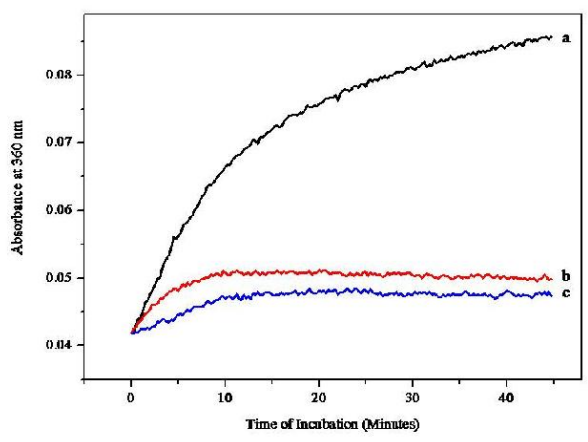

Figure 8D. SHSP19.9 (Pre-incubated for $30 \mathrm{~min}$ at $60^{\circ} \mathrm{C}$ ) with $10 \mathrm{mM} \mathrm{DTT}$ against BLC aggregation at 1:1 molar ratio, where (a) represents BLC only, (b) and (c) demonstrate without and with preincubated SHSP19 respectively.

Thus pre-incubation of sHSP20.8 before applying against BLC aggregation bear some extent of successful difference compared with the without pre-incubated SHSP20.8. It is assumed that during incubation, BLC took some times to be dissociated and within this time sHSP20.8 was converted to its active species (large oligomer) and both of the species were assumed to be essential for proper interaction with each other to form a large, defined chaperone-substrate complex (Haslbeck et al., 
1999), which can also be evaluated by SDSPAGE (Haslbeck et al., 1999). So, when sHSP20.8 and SHSP19.9 were pre-incubated at $60^{\circ} \mathrm{C}$ for a certain time before incorporating of BLC must facilitates SHSPs to be dissociated into large oligomer (active species), which are very essential for successful interaction to form noble complex between SHSPs and BLC. This is resulting the suppression of thermal induced aggregation of BLC very efficiently. Such activation also coincides with dissociation of the HSP26 oligomers into dimers, leading to a proposal of the dissociation mediated activation mechanism. In fact the yeast HSP26 becomes activated at elevated temperatures and induces the dissociation of the oligomeric complex into dimers and these dimers initiate the interaction with nonnative substrate proteins and reassemble into larger well-defined HSP26nonnative substrate protein complex (Haslbeck et al., 1999, Haslbeck et al., 2005). Interaction between active species of SHSPs and intact BLC is not possible. So the findings for preincubated SHSPs and without pre-incubated sHSPs are observed to be different and in other words, pre-incubation is an effective method to accelerate the chaperone function of silkworm sHSPs. When both the sHSPs and BLC were applied at the same time, both took some time (lag phase) to be converted into active species. But the lag phase for BLC and the examined sHSPs are different. Silkworm sHSPs might take less time to be converted into its active species than the non-native substrate protein BLC (5 minutes). As soon as BLC is dissociated into dimer it interacts with large oligomeric sHSPs and formed the SHSP-BLC complex and aggregation of BLC is prevented. Because if the lag phase of silkworm sHSPs would more than the non-native protein, the protein would be aggregated and it could not be recovered.

Moreover, sHSPs can prevent the further aggregation of BLC only, but cannot solubilizes the already-aggregated BLC as like as $\beta$-casein against insulin (Zhang et al., 2005), which is an agreement of the activity of SHSP20.8. It is seen that pre-incubation converts the SHSPs to the active species prior to the activation of nonnative protein and thus can overcome the problem. These results unambiguously demonstrate that SHSP20.8 does exhibit a CLA when analyzed in such in vitro system. A similar phenomenon was also happened in the case of sHSP19.9 in the presence of DTT and so the pre-incubation of SHSP19.9 bear satisfactory performance against the thermal aggregation of BLC.

\section{Acknowledgement}

This work was conduct with the approval of the KEK-PF Proposal Review Committee (Proposal No. 2007G665). It was partially supported by the financial aid from the Japan Foundation for Applied Enzymology. It was partially supported by the National Bio-Resources Project (Silkworm) of the Ministry of Education, Culture, Sports, Science and Technology, Japan.

\section{Conclusion}

CLA of pre-incubated silkworm sHSP19.9 and SHSP20.8 was observed against BLC aggregation beside control (without preincubated). Pre-incubation of the selected proteins were found to provide reproducible effects to a considerable extent. So, it can be concluded that pre-incubation is an accelerating factor to enhance the CLA of SHSP19.9 and sHSP20.8.

\section{References}

Abdulle R, A Mohindra, P Fernando and JJ Heikkila (2002). Xenopus small heat shock proteins, Hsp30C and Hsp30D, maintain heat- and chemically denatured luciferase in a foldingcompetent state. Cell Stress Chaperone 7:616.

Arrigo AP (2005). In search of the molecular mechanism by which small stress proteins counteract apoptosis during cellular differentiation. Journal of Cell Biochemistry 94:241-246.

Aso Y, D Sakano, B Li, QY Xia and H Fuji (2006). Small heat shock proteins of the silkworm, Bombyx mori. $5^{\text {th }}$ International Symposium on Molecular Insect Science, Tuscon, AZ, p.22.

Basha E, KL Friedrich and E Vierling (2006). The Nterminal arm of small heat shock proteins is important for both chaperone activity and substrate specificity. Journal of Biological Chemistry 281:39943-39952.

Bova MP, Q Huang, L Din and J Horwitz (2002). Subunit exchange, conformational stability, and chaperone-like function of the small heat shock protein 16.5 from Methanococcus jannaschii. Journal of Biological Chemistry 277:38468-38475.

Chowdary TK, B Raman, T Ramakrishna and CM Rao (2004). Mammalian Hsp22 is a heatinducible small heat shock protein with chaperone-like activity. Biochemistry Journal 381:379-387.

Giese KC and E Veirling (2002). Changes in oligomerization are essential for the chaperone activity of a small heat shock protein in vivo and in vitro. Journal of Biological Chemistry 277:46310-46318.

Haslbeck M, N Braun, T Stromer, B Richter, N Model, S Weinkauf and J Buchner (2004). 
Hsp42 is the general small heat shock protein in the cytosol of Saccharomyces cerevisiae. EMBO Journal 23:638-649

Haslbeck M, T Franzmann, D Weinfurtner and J Bucher (2005). Some like it hot: the structure and function of small heat-shock proteins. Nature Structural \& Molecular Biology 12: 842-846.

Haslbeck M, S Walke, T Stromer, M Ehrnsperger, HE White, S Chen, HR Saibil and J Buchner (1999). Hsp26: a temperature-regulated chaperone. EMBO Journal 18:6744-6751.

Hook DWA and JJ Harding (1997). Molecular chaperone protect catalase against thermal stress. European Journal of Biochemistry 247:380-385.

Horwich AL (2002). Protein aggregation in disease: a role for folding intermediates forming specific multimeric interactions. Journal of Clinical Investigation 110:1221-1232.

Hossain MT and Y Aso (2017). Thermally induced aggregation and its suppression of silkworm small heat shock protein sHSP19.9. Bangladesh Journal of Animal Science 46 (1):57-64.

Hossain MT, S Teshiba, Y Shigeoka, T Fujisawa, Y Inoko, D Sakano, $\mathrm{K}$ Yamamoto, $Y$ Banno and $Y$ Aso (2010). Structural Properties of Silkworm Small Heat Shock Proteins: SHSP19.9 and sHSP20.8. Bioscience Biotechnology and Biochemistry 74(8):1556-1563.

Kaldis A, BG Atkinson, JJ Heikkila (2004). Molecular chaperone function of the Rana catesbeiana small heat shock protein, hsp30. 2004 Comparative Biochemistry and Physiology Part A: Molecular \& Integrative Physiology 139(2):175-82.

Lee GJ and E Vierling (2000). A small heat shock protein cooperates with heat shock protein 70 systems to reactivate a heat-denatured protein. Plant Physiology 122:189-198.

Manna T, T Sarkar, A Poddar, M Roychowdhury, KP Das and B Bhattacharyya (2001). CLA of tubulin. Journal of Biological Chemistry 276:39742-39747.

Mao Q, D Ke, X Feng and Z Chang (2001). Preheat treatment for Mycobacterium tuberculosis Hsp16.3: correlation between a structural phase change at $60^{\circ} \mathrm{C}$ and a dramatic increase in chaperone-like activity. Biochemistry and Biophysical Research Communication 284:942-947.
Morrow G, JJ Heikkila and RM Tagnuay (2006). Differences in the chaperone-like activities of the four main small heat shock proteins of Drosophila melanogaster. Cell Stress \& Chaperone 11(1):51-60.

Morrow G, M Samson, S Michaud and RM Tanguay (2004). Over expression of the small mitochondrial Hsp22 extends drosophila life span and increases resistance to oxidative stress. FASEB Journal 18:598-599.

Panensenko OO, A Seit Nebi, OV Bukach, SB Marston and NB Gusev (2002). Structure and properties of avian small heat shock protein with molecular weight $25 \mathrm{kDa}$. Biochemical Biophysical Acta 1601:64-74.

Sakano D, B Li, QY Xia, K Yamamoto, Y Banno, H Fuji and $Y$ Aso (2006). Gene encoding small heat shock proteins of silkworm, Bombyx mori. Bioscience Biotechnology and Biochemistry 70:2443-2450.

Smýkal P, J Mašín, I Hrdý, I Konopásek and V Žárský (2000). Chaperone activity of tobacco HSP18, a small heat-shock protein, is inhibited by ATP. Plant Journal 23:703-713.

van Montfort RLM, E Basha, KL Friedrich, C Slingsby and E Vierling (2001). Crystal structure and assembly of a eukaryotic small heat shock protein. Nature Structural Biology 8:1025-1030.

van Montfort R, C Slingsby and E Vierling (2002). Structure and function of the small heat shock protein/a-crystallin family of molecular chaperones. Advance Protein Chemistry 59:105-156.

Wang K and BI Kurganov (2003). Kinetics of heatand acidification-induced aggregation of firefly luciferase. Biophysical Chemistry 106:97-109.

Xinmiao $F$, L Chong, $L$ Yang, $F$ Xiuguang, $G$ Liangcai, C Xiaoyou and C Zengyi (2003). Small heat shock protein Hsp16.3 modulates its chaperone activity by adjusting the rate of oligomeric dissociation. Biochemical and Biophysical Research Communication 310:412-420.

Zhang $\mathrm{X}, \mathrm{X}$ Fu, $\mathrm{H}$ Zhang, $\mathrm{C}$ Liu, $\mathrm{W}$ Jiao and $\mathrm{Z}$ Chang (2005). Chaperone-like activity of $\beta$ casein. International Journal of Biochemistry and Cell Biology 37:1232-40. 